\title{
Investigation of Morphology and Chemical Composition of Self-Organized Semiconductor Quantum Dots and Wires by X-Ray Scattering
}

\author{
V. HOLÝ* $\hat{Y}^{*}$ M. MEDUŇA \\ Institute of Condensed Matter Physics, Masaryk University \\ Brno, Czech Republic
}

J. Stangl, T. Roch And G. Bauer

Institute of Semiconductor Physics, Kepler University, Linz, Austria

X-ray scattering methods suitable for the investigation of the morphology and chemical composition of self-organized quantum dots and quantum wires are reviewed. Their application is demonstrated in experimental examples showing that a combination of small angle $\mathrm{X}$-ray scattering with high-resolution $\mathrm{X}$-ray diffraction can reveal both the shape and the chemical composition of the self-organized objects.

PACS numbers: 61.10.-i, 61.10.Dp

\section{Introduction}

The spontaneous formation of self-organized islands and ripples at interfaces of lattice mismatched semiconductor superlattices has been recently developed as a promising approach for the fabrication of quantum dots and wires [1]. Quasi-zero-dimensional self-organized quantum dots (SQD) are grown usually in the Stranski-Krastanow epitaxial growth mode. The driving force for this process is the elastic relaxation of internal stresses accompanied by a lateral compression or expansion of the dots. Unfortunately, these self-organized dots are usually not very uniform in size and spacing, which limits seriously their applicability. The growth of superlattices is a possible way for improving the uniformity of the dots

*corresponding author; e-mail: holy@physics.muni.cz 
$[2,3]$. Vertical ordering of the dots caused by inhomogeneous elastic strain fields gives rise also to a lateral ordering and a size homogenization.

In different materials, different types of ordering occurs, for instance vertical dot columns occur in InAs/GaAs [4] and SiGe/Si systems [5], in the superlattices of type IV-VI a trigonal dot arrangement has been observed [6, 7]. The different arrangements can be explained quantitatively using the idea of the island nucleation in the minima of the local elastic energy density [2, 7]. In samples of $\mathrm{PbSe} / \mathrm{PbEuTe}$ we have observed previously a transition from a vertical dot stacking (for smallest superlattice periods) via trigonal-type stacking (moderate periods) to completely uncorrelated dot positions (for larger superlattice periods) [7, 8]. A tetragonal dot stacking was observed in $\mathrm{CdZnSe/ZnSe} \mathrm{superlattices} \mathrm{[9].}$

Much less work has been devoted to the investigation of one-dimensional self-organized quantum wires (SQW) [10-12]. It was shown that the growth of such wires requires a suitable template, which can be provided, e.g., by regular arrays of terraces formed during a step-bunching growth process [13-15]. Such step-bunching instabilities were observed to occur in several systems, including Si/SiGe multilayers grown on miscut Si substrates. Brunner et al. [16] have shown that for molecular beam epitaxy (MBE) growth of SiGe nanostructures, certain ranges of growth parameters exist, where either laterally ordered SiGe island growth takes place, or wires are formed on nearly equally spaced terraces, which were studied by transmission electron microscopy (TEM) and atomic force microscopy (AFM).

The shape and the arrangement of the self-assembled quantum dots and wires can be studied directly by AFM and by TEM. Both methods have serious limitations; the former method is limited only to the topmost free-standing dot layer, the latter one requires an elaborate sample preparation. An indirect way for studying the morphology of the SQD and SQW consists in small angle X-ray scattering. This non-destructive method can investigate both the free-standing and buried objects, and in contrast to TEM and X-ray diffraction, it is not sensitive to elastic deformation.

A small-angle X-ray scattering experiment yields a distribution of the scattered intensity in reciprocal space. In the usual coplanar arrangement, the wave vectors $\boldsymbol{K}_{\mathrm{i}, \mathrm{f}}$ of the primary and scattered beam, as well as the surface normal of the sample lie in the same plane (scattering plane). In this arrangement, the accessible part of reciprocal space is limited by the condition $\alpha_{\mathrm{i}, \mathrm{f}}>0$, where $\alpha_{\mathrm{i}, \mathrm{f}}$ are the angles of incidence and exit of the radiation with respect to the sample surface. Thus, for a given scattering angle $2 \Theta=\alpha_{\mathrm{i}}+\alpha_{\mathrm{f}}$, one can detect the objects of a minimum characteristic size and/or distance

$$
L_{\min }>\frac{\lambda}{2 \Theta^{2}} .
$$

Since the maximum value of $\Theta$ is limited by the intensity of scattering to few degrees, this condition represents a substantial limitation of the application of the coplanar arrangement. 
In grazing-incidence small-angle X-ray scattering (GISAXS) geometry, the plane of the vectors $\boldsymbol{K}_{\mathrm{i}, \mathrm{f}}$ is nearly parallel to the sample surface and no limitation of the dot size exists [17]. In addition, changing the incidence and/or exit angles $\alpha_{i, f}$ we can tune the depth, where the scattered radiation comes from (the information depth).

High-resolution X-ray scattering is sensitive mainly to the strain fields in the objects and around them. In most cases, the volume of the objects under investigation is very small and therefore a conventional coplanar X-ray diffraction (XRD) is not suitable. Instead, grazing-incidence diffraction (GID) must be applied. In this geometry, the diffracting net plane is nearly perpendicular to the sample surface and the wave vectors $\boldsymbol{K}_{\mathrm{i}, \mathrm{f}}>0$ are almost parallel to it. Similarly to GISAXS, the information depth can be tuned in the GID method.

In the first part of this paper we describe briefly the mechanism of X-ray scattering from self-organized objects, in the second part we show two experimental examples of the application of the scattering methods.

\section{Theoretical description}

The theoretical description of both small-angle scattering (GISAXS) and high-angle diffraction (GID) can be formulated in the uniform way using the distorted-wave Born approximation (DWBA) $[18,19]$. In this method, the scattering potential $V(r)$ occurring in the wave equation

$$
\left(\Delta+K^{2}\right) E(r)=V(\boldsymbol{r}) E(r)
$$

is divided into two parts

$$
V(r)=V_{A}(\boldsymbol{r})+V_{B}(r)
$$

where the first part describes the scattering from a non-disturbed system (an amorphous semi-infinite substrate in our case) and the second part accounts for the scattering from the objects. In the first step of the calculation procedure, we find two exact independent solutions of the wave equation of the undisturbed system

$$
\left(\Delta+K^{2}\right) E_{A}(r)=V_{A}(r) E_{A}(r)
$$

denoted $E_{A}^{(1,2)}$ (see Fig. 1). The incident wave of the solution $E_{A}^{(1)}, \boldsymbol{K}_{\mathrm{i}}^{(1)} \equiv \boldsymbol{K}_{\mathrm{i}}$, corresponds to the actual incoming beam, the solution $E_{A}^{(2)}$ is time-inverted and
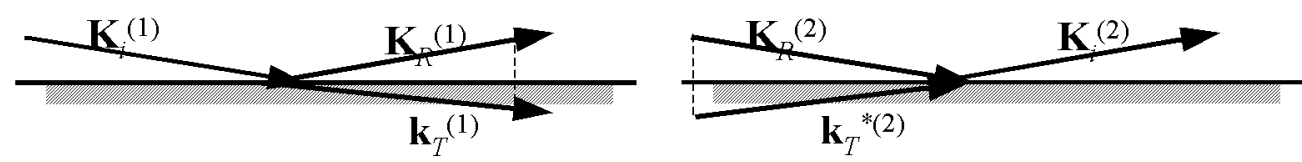

Fig. 1. Sketch of the solutions of the undisturbed wave equation (4). 
its wave $\boldsymbol{K}_{\mathrm{i}}^{(2)} \equiv \boldsymbol{K}_{\mathrm{f}}$ is the true scattered wave. The scattered intensity is usually expressed in reciprocal space as a function of the scattering vector $\boldsymbol{Q}=\boldsymbol{K}_{\mathrm{f}}-\boldsymbol{K}_{\mathrm{i}}$. For the chosen undisturbed system, the solution of Eq. (4) is trivial and it can be represented by the Fresnel transmission and reflection coefficients.

In the second step, we use these solutions for the calculation of the differential cross-section of X-ray scattering due to the disturbance $V_{B}(\boldsymbol{r})$. In most cases, this scattering potential is random and we have to a verage the differential cross-section over all macroscopically non-resolvable configurations of the objects. The a veraged cross-section consists in two contributions. The coherent contribution represents the scattering due to $V_{A}(r)+\left\langle V_{B}(r)\right\rangle$, the intensity $I_{\mathrm{coh}}(\boldsymbol{Q})$ of this contribution is concentrated in reciprocal space along the normal to the surface (crystal truncation rod) crossing the lattice point of the crystal reciprocal lattice. In the following, we will not deal with the coherent component and we will restrict ourselves only to the incoherent (diffuse) component, whose intensity $I(\boldsymbol{Q})$ is distributed in a vicinity of the truncation rod. The differential cross-section of the diffuse scattering is

$$
\left(\frac{\mathrm{d} \sigma}{\mathrm{d} \omega}\right)_{\mathrm{diff}}=\frac{1}{16 \pi^{2}}\left(\left\langle|W|^{2}\right\rangle-|\langle W\rangle|^{2}\right),
$$

where

$$
W=\left\langle E_{A}^{(2)}\left|V_{B}\right| E_{A}^{(1)}\right\rangle
$$

is the scattering matrix element. Inserting the corresponding expressions for the undisturbed wave fields $E_{A}^{(1,2)}$ we express this matrix element as a sum over several scattering processes. If, for instance, the objects are located at the sample surface, the matrix element consists in 4 scattering processes, sketched schematically in Fig. 2.
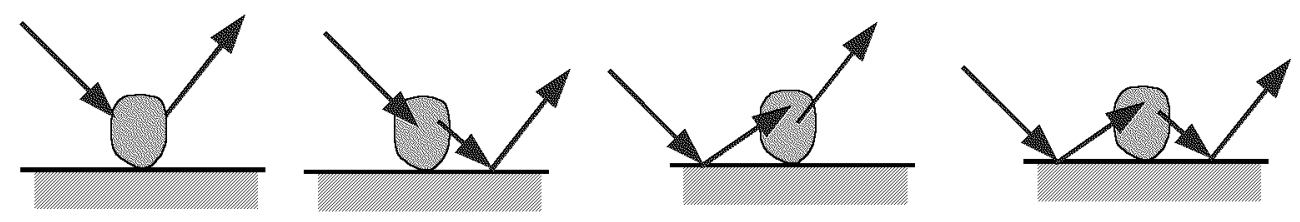

Fig. 2. Scattering processes considered in the calculation in the case of free-standing objects. The second process, for instance, includes the scattering of the incident wave from the object and the reflection of the scattered wave from the sample surface.

In the small-angle scattering method, the disturbance $V_{B}(\boldsymbol{r})$ comprises only the contrast $\Delta \chi(r)$ in the crystal polarizability of the object and its neighborhood, i.e. it is sensitive mainly to the shape of the objects

$$
V_{B}(r)=K^{2} \Delta \chi \Omega(r)
$$

where $\Omega(r)$ is the shape function of the objects (unity in the objects and zero outside). In GID however, the disturbance $V_{B}(r)$ includes also the elastic strains in the object and around it 


$$
V_{B}(r)=K^{2}\left[\Delta \chi \Omega(r) e^{-\mathrm{i} /{ }^{\prime} \cdot u(\boldsymbol{r})}-\chi_{\text {matrix }}\right]
$$

Here we have denoted as $\chi_{\text {matrix }}$ the crystal polarizability of the host lattice, $u(r)$ is the displacement field due to the objects and $h$ is the reciprocal lattice vector (diffraction vector).

The resulting formula for the scattered intensity can be simplified substantially if we assume that all the objects are identical. Then

$$
\left(\frac{\mathrm{d} \sigma}{\mathrm{d} \omega}\right)_{\mathrm{diff}}=\mathrm{const} \sum_{p}\left|A_{p} f\left(\boldsymbol{Q}_{p}\right)\right|^{2} G\left(\boldsymbol{Q}_{p}\right),
$$

where the sum runs over all active scattering processes,

$$
G(\boldsymbol{Q})=\left\langle\sum_{n, m} \mathrm{e}^{-\mathrm{i}\left(\boldsymbol{Q} \cdot R_{n}-\boldsymbol{Q}^{*} \cdot R m\right)}\right\rangle
$$

is the correlation function of the positions of the objects, $A_{p}$ is the amplitude of the $p$-th process and $F(\boldsymbol{Q})$ is the structure factor of a single object. In GISAXS, this structure factor is proportional to the Fourier transformation of the shape function of a single object

$$
F(\boldsymbol{Q})=\mathrm{FT}\left[\Delta \chi \Omega_{\mathrm{obj}}(r)\right]
$$

In XRD and GID however, the expression of the structure factor is more complicated

$$
F(\boldsymbol{Q})=\mathbf{F T}\left[\chi_{\text {matrix }}\left(\mathrm{e}^{-\mathrm{i} \not \boldsymbol{h} \cdot u_{\mathrm{obj}}(\boldsymbol{r})}-1\right)+\Delta \chi \Omega_{d}(r) \mathrm{e}^{-\mathrm{i} \hbar \cdot u_{\mathrm{obj}}(\boldsymbol{r})}\right]
$$

containing also the displacement field $u_{\mathrm{obj}}(r)$ of a single object.

Due to the correlations of the object positions expressed in the correlation function (9), the scattered intensity is concentrated to a sequence of maxima in reciprocal space (intensity satellites). The positions of these maxima correspond to the points of the lattice reciprocal to the "object lattice" and their width is determined by the degree of ordering of the object positions. For a perfectly periodic object arrangement the intensity satellites are infinitely narrow. The heights of the satellites are given by $|F(\boldsymbol{Q})|^{2}$.

\section{Experimental examples}

In this section we present results of X-ray scattering investigation of self-assembled PbSe quantum dots in $\mathrm{PbSe} / \mathrm{PbEuTe}$ superlattices and self-assembled SiGe quantum wires in $\mathrm{SiGe/Si} \mathrm{superlattices.}$

\subsection{PbSe quantum dots in PbSe/PbEuTe superlattices}

The samples were grown by molecular beam epitaxy on PbTe buffer layers deposited on (111) $\mathrm{BaF}_{2}$ cleaved substrates. All samples consisted of 5 monolayers $\mathrm{PbSe}$ and spacer layers $\mathrm{Pb}_{1-x} \mathrm{Eu}_{x}$ Te with the superlattice periods $D$ ranging from 
350 to $650 \AA$. Because of the $5.4 \%$ lattice mismatch of PbSe with respect to $\mathrm{PbTe}$, island growth in the Stranski-Krastanow growth mode occurs once the critical thickness of 1.5 monolayers is exceeded. During the overgrowth of the islands, a rapid replanarization occurs and a completely flat surface is regained after the growth of $200 \AA \mathrm{PbEuTe}$. The concentration $x$ of Eu was adjusted in order to achieve a complete strain symmetrization of the superlattice period with respect to the $\mathrm{PbTe}$ buffer. The strain symmetrization prevents plastic relaxation via misfit dislocations. For this study, we have used three superlattice samples with different types of the dot stacking, the parameters of the samples are summarized in Table $\mathbf{I}$, other details of the growth procedure can be found elsewhere [20].

TABLE I

Basic structural parameters of the $\mathrm{PbSe} / \mathrm{PbEuTe}$ superlattices.

\begin{tabular}{c|c|c|c}
\hline \hline Sample no. & $D[\AA]$ & $\begin{array}{c}\text { Number of periods } \\
N\end{array}$ & Stacking type \\
\hline 966 & 350 & 30 & vertical \\
925 & 470 & 60 & trigonal \\
906 & 660 & 60 & random
\end{tabular}

From TEM and AFM studies it follows that the free-standing quantum dots have the shape of threefold pyramid with well developed side $\{001\}$ facets. The shape of the buried dots could not be reliably resolved from TEM pictures, since the contrast in TEM is mainly affected by the strain fields around the dots and not by the dot shapes.

The GISAXS measurements have been performed at the TROIKA II beamline at ESRF, Grenoble using the wavelength $1.5545 \AA$. We have measured the distribution of the scattered intensity in several $\left(Q_{x} Q_{y}\right)$ planes parallel with the sample surface, the examples of the results are shown in Fig. 3. It can be seen in this figure that the dots are laterally not ordered in samples 966 and 906 , while a trigonal ordering is visible in sample 925 . The vertical ordering of the dots (i.e. in direction perpendicular to the sample surface) cannot be established from these maps, other measurements not shown here revealed a perfect vertical ordering in sample 966 and no ordering of the dot positions in 906.

From these intensity distributions we have extracted line scans along the arrows in Fig. 3 and we have compared these scans with numerical simulations using Eqs. (8)-(10). The resulting scans are plotted in Fig. 4. In the simulations we have assumed that the buried dots have the shape of a truncated threefold pyramid with rounded edges. The form of the pyramid base was expressed using the formula 

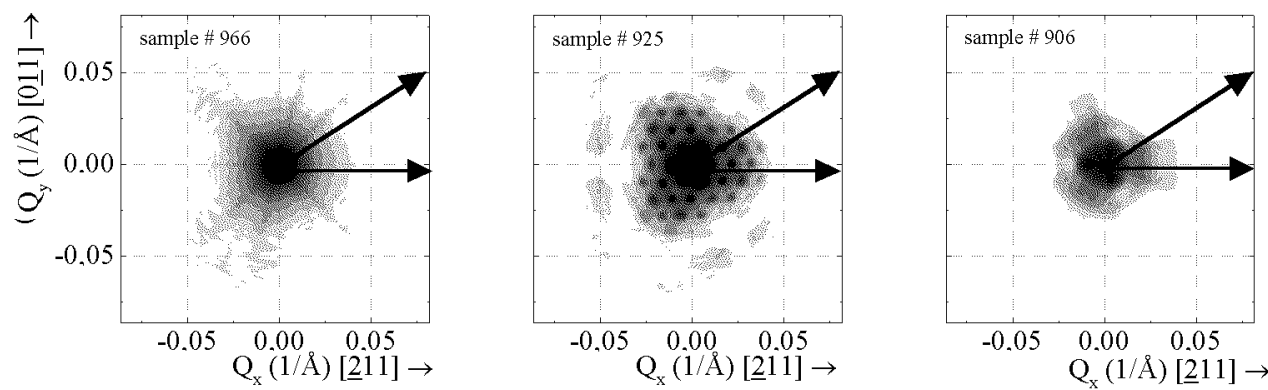

Fig. 3. Distribution of the scattered intensity in a $\left(Q_{x} Q_{y}\right)$ plane parallel with the sample surface, GISAXS geometry. The arrows denote the directions of the extracted line scans corresponding to the azimuthal angles $\phi=0^{\circ}$ and $30^{\circ}$.

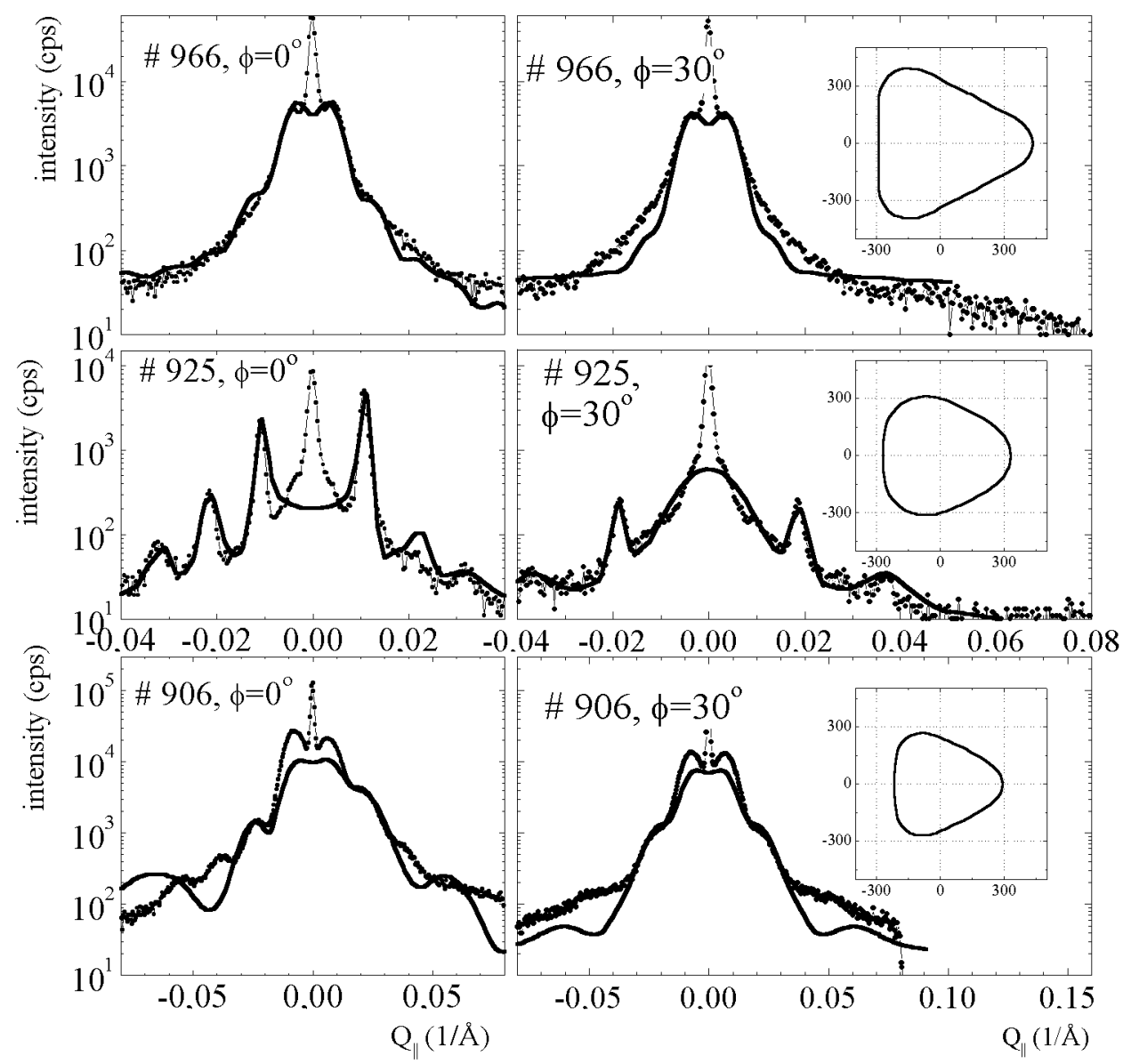

Fig. 4. The linear scans extracted from the GISAXS intensity maps shown in Fig. 3 (points) and their theoretical simulations (lines). The shapes of the dot base following from the fits are shown in the insets. 


$$
R(\phi)=\sum_{m=0}^{\infty} R_{m} \cos (3 m \phi)
$$

in polar coordinates, from the fit we have determined the coefficients $R_{0,1,2}$. The resulting shapes of the dot base are shown in the insets in Fig. 4. From the fit we have also determined the relative height $h_{\text {rel }}$ of the pyramid with respect to a non-truncated sharp pyramid and the mean distance $\langle L\rangle$ of the neighboring dots, the details have been published elsewhere [21]. The results are summarized in Table II.

TABLE II

Parameters of the $\mathrm{PbSe}$ dots in the $\mathrm{PbSe} / \mathrm{PbEuTe}$ superlattices determined from the GISAXS data.

\begin{tabular}{c|c|c|c|c|c|c}
\hline \hline $\begin{array}{c}\text { Sample } \\
\text { no. }\end{array}$ & $D[\AA]$ & $\langle L\rangle[\AA]$ & $h_{\text {rel }}$ & $R_{0}[\AA]$ & $R_{1} / R_{0}$ & $R_{2} / R_{0}$ \\
\hline 966 & 350 & $1050 \pm 150$ & $0.5 \pm 0.2$ & $380 \pm 50$ & $0.2 \pm 0.05$ & $0.04 \pm 0.02$ \\
925 & 470 & $680 \pm 20$ & $0.3 \pm 0.1$ & $300 \pm 60$ & $0.1 \pm 0.05$ & \\
960 & 660 & $550 \pm 100$ & $0.5 \pm 0.2$ & $250 \pm 70$ & $0.15 \pm 0.05$ & $0.02 \pm 0.01$
\end{tabular}

From the data it follows that the shape of the buried dots differs substantially from that of the free-standing ones, most likely due to interdiffusion. X-ray scattering is the only method that can reveal the shape of these buried quantum dots. In order to study the chemical composition of the dots, we have used coplanar XRD. Figure 5 shows the distribution of the diffracted intensity in reciprocal $\left(Q_{x} Q_{z}\right)$ plane perpendicular to the sample surface close to the reciprocal lattice point 111. The intensity maps have been measured using the wavelength $1.54 \AA$ at the beamlines TROIKA II and ROBL at ESRF, Grenoble. The maps have been plotted in the coordinates of the reduced scattering vector

$$
q=Q-h
$$

The type of the stacking of the dots clearly follows from the intensity maps. The dots in sample 966 are correlated only vertically and no horizontal ordering is present. Therefore, the scattered intensity is concentrated in horizontal sheets and the vertical width of the sheets is inversely proportional to the vertical correlation length of the dot positions. In sample 925 a full three-dimensional trigonal dot arrangement is present. Therefore, the intensity distribution in reciprocal space has maxima in lattice points in the reciprocal "dot lattice" and the maxima in the intensity map correspond to the cross-section of the (01-1) scattering plane with this dot lattice. No correlation of the dot positions is present in sample 906 . The correlation function $G(\boldsymbol{Q})$ is constant in this case and the intensity distribution is proportional to that of a single dot given by $\left|F_{p}(\boldsymbol{Q})\right|^{2}$. 

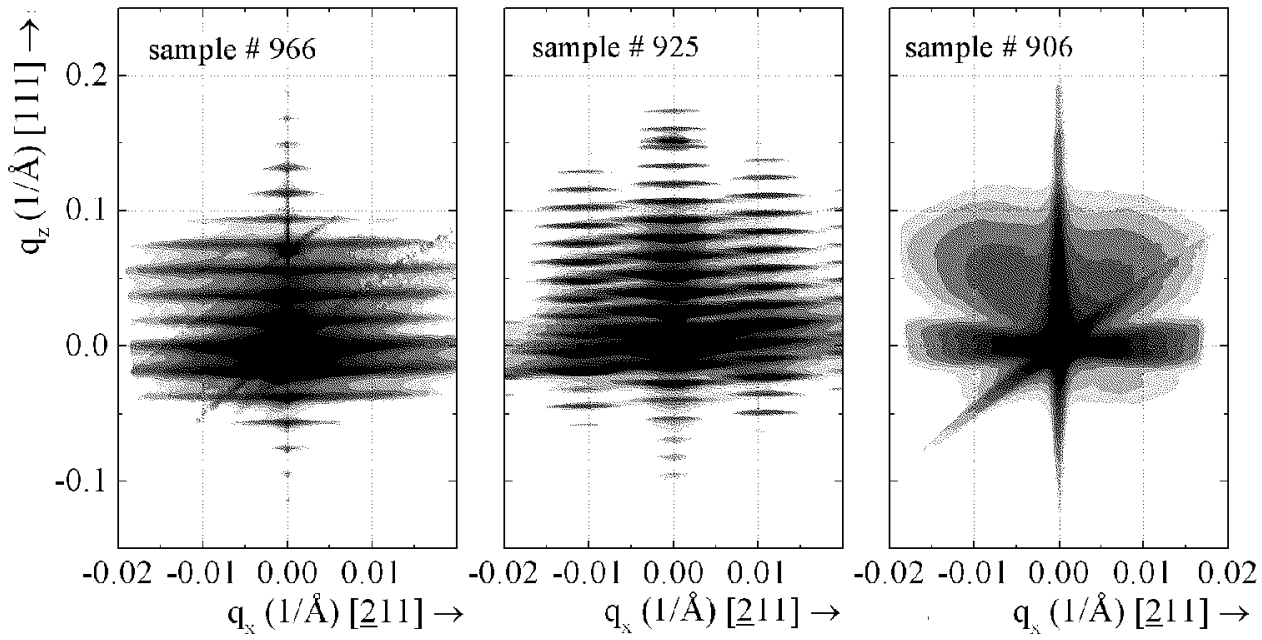

Fig. 5. XRD intensity maps of $\mathrm{PbSe} / \mathrm{PbEuTe}$ superlattices, symmetrical diffraction 111.

From the map of sample 925 we have extracted two linear scans parallel to the $Q_{z}$ axis crossing the first and the second lateral intensity satellites and we have compared them with the simulations using Eqs. (8), (9), (11) (Fig. 6).

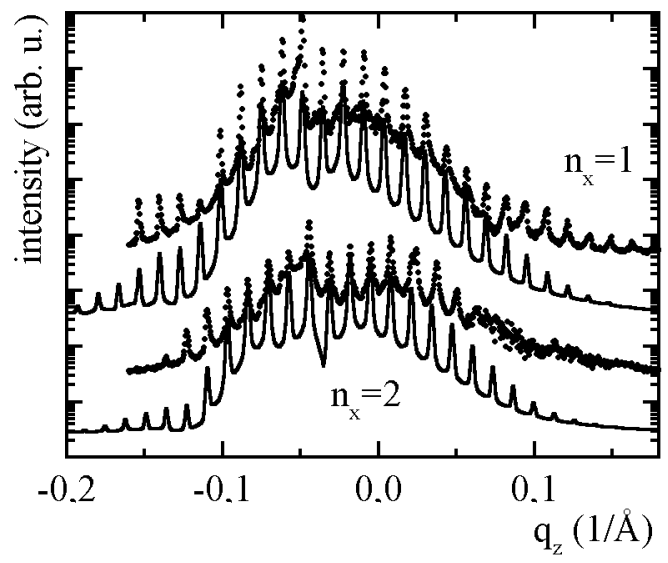

Fig. 6. The linear scans extracted from the intensity map of sample 925 (Fig. 5, points) and their theoretical simulations (lines). The experimental curves are shifted upwards for clarity.

In the simulations we used the dot shape following from the GISAXS data, the only free parameter was the chemical composition of the dots. The best correspondence of the measured data with theory could be achieved assuming the composition of the dot lattice $\mathrm{PbSe}_{0.5} \mathrm{Te}_{0.5}$. Therefore, interdiffusion affects not only the shape but also the chemical composition of the buried quantum dots. 


\subsection{SiGe self-assembled quantum wires in a SiGe/Si superlattice}

We have studied a 20 period $\mathrm{SiGe/Si} \mathrm{multilayer,} \mathrm{grown} \mathrm{by} \mathrm{solid} \mathrm{source} \mathrm{MBE}$ at the Technical University Munich (Prof. G. Abstreiter) on a [001]-oriented Si substrate with a miscut of $3.5^{\circ}$ towards the [100] direction. With the chosen growth conditions, a smooth $\mathrm{Si}$ buffer was grown. The thickness of the $\mathrm{Si}_{0.55} \mathrm{Ge}_{0.45}$ and Si layers was nominally $2.5 \mathrm{~nm}$ and $10 \mathrm{~nm}$, respectively. The whole multilayer stack was covered by a $12 \mathrm{~nm}$ thick Si capping layer. The substrate temperature during multilayer growth was $550^{\circ} \mathrm{C}$, for growth details see [16]. In AFM pictures of the surface we can resolve a one-dimensional ripple pattern, the ripples are perpendicular to the miscut direction [100]. The surface ripples correspond to one-dimensional quantum SiGe wires buried at the SiGe/Si interfaces below the surface.

The GISAXS and GID experiments were performed at the TROIKA II beamline at ESRF using the wavelength $1.55 \AA$. Figure 7 shows the measured intensity distribution in reciprocal space along the line perpendicular to the wires for various $Q_{z}$.

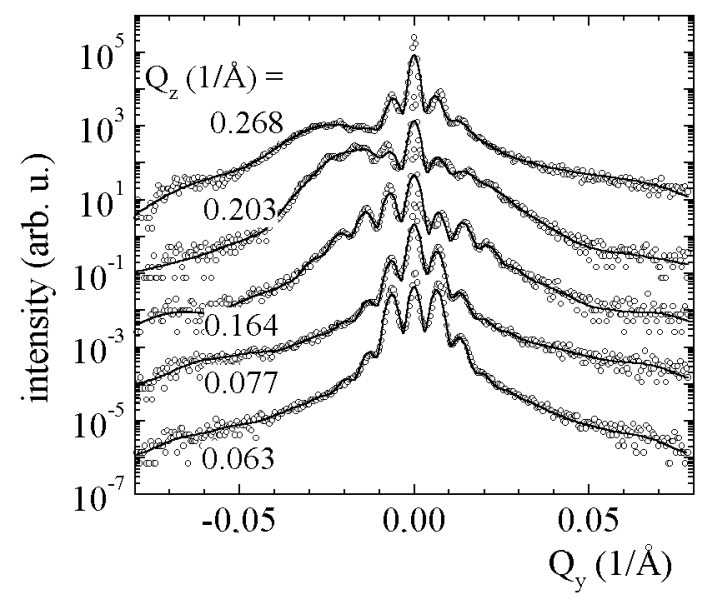

Fig. 7. GISAXS scans measured across the SiGe wires in a SiGe/Si superlattice (points) and their fits (lines).

The lateral ordering of the wire positions gives rise to intensity maxima. From their distances and widths we determined the mean wire distance (900 \pm 50$) \AA$ and the root mean square deviation of wire positions $(150 \pm 50) \AA$, therefore, the wire positions are correlated up to the 6 th neighbor. The heights of the satellites depend on the shape of the wire cross-section. From the numerical analysis it follows that the wires have a triangular shape, the slope of their side walls is about $6^{\circ}$ (Fig. 8) [22]. 


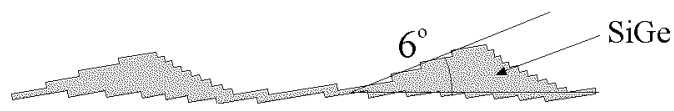

Fig. 8. The shape of the cross-section of the SiGe wire.

This finding indicates that the wires could not be created only by a bunching of the monolayer steps at a vicinal growing surface, since the bunching process could produce the wires with the slope of the side walls smaller than or equal to the miscut $\left(3.5^{\circ}\right.$ in our case).

The chemical composition of the wires has been studied by means of the GID method. We have chosen 400 diffraction, the diffraction vector was perpendicular to the wires. In this diffraction we have recorded a distribution of the scattered intensity in $\left(Q_{x} Q_{z}\right)$ plane perpendicular to the sample surface, the $Q_{x}$ axis is parallel to the diffraction vector $\boldsymbol{h}=[400]$. The intensity map is shown in Fig. 9 . The periodicity of the lateral wire positions gave rise to lateral satellites and the vertical superlattice periodicity caused a vertical modulation of these satellites. Since the replication of the positions of the wires at different interfaces occurred in a direction inclined by the angle of about $5^{\circ}$ from the growth direction, the
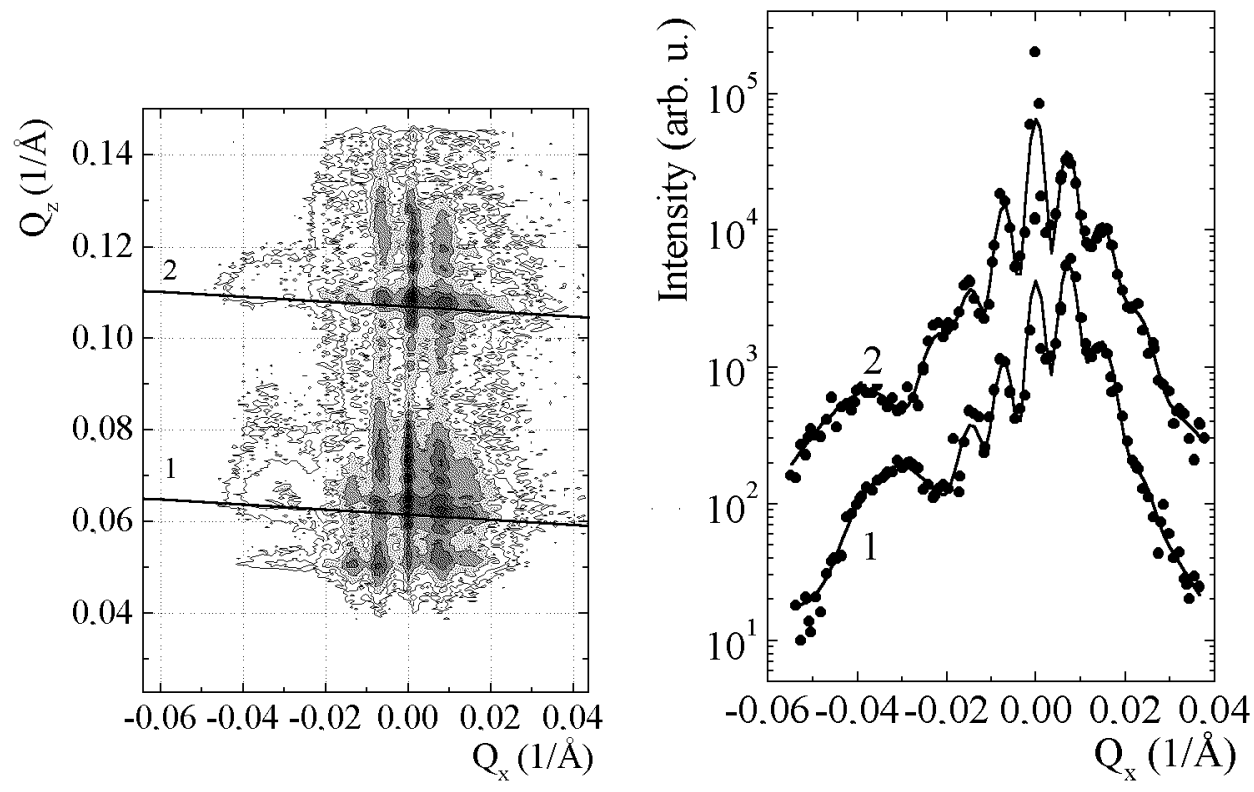

Fig. 9. The reciprocal space map of a SiGe/Si multilayer taken in the GID geometry, 400 diffraction (left) and the linear scans extracted from the map along the lines 1 and 2 (right, points). The full lines in the right panel denote the simulations. 
maxima at different lateral satellites are arranged in intensity "sheets" making the angle $5^{\circ}$ with the horizontal direction. These sheets are denoted by 1 and 2 in Fig. 9. From the maps we have extracted linear intensity scans along the sheets (Fig. 9, right panel) and we have simulated them using Eqs. (8), (9), (11). In the simulations, we took the shape of the wires from the GISAXS measurements and we have adjusted only the Ge concentration $x$ in the wires as a single free parameter. From the fit $x=(20 \pm 10) \%$ follows, so that the Ge content in the buried wires is much smaller than the nominal value $45 \%$.

\section{Summary}

We have demonstrated that the shape, position and chemical composition of self-assembled quantum dots and wires in semiconductor superlattices can be studied by surface-sensitive GISAXS and GID. The GISAXS method is sensitive mainly to the shape and position of the self-assembled objects, while GID is affected by the strain field in these objects and around them caused by the mutual lattice mismatch. Since the self-assembled objects are extremely small, the scattered intensities are usually very weak. Therefore, the X-ray experiments can be performed only using a synchrotron radiation.

\section{Acknowledgment}

The work has been supported by the Grant Agency of Czech Republic (project 202/00/0354), by the Ministry of Education of Czech Republic (project VS 96102) and by FWF Vienna, Austria. We acknowledge the assistance of O. Konovalov and D. Smilgies (ESRF Grenoble) with the beam line setup. The SiGe/Si multilayers have been grown at Technical University of Munich (Germany) by G. Abstreiter.

\section{References}

[1] D. Leonard, M. Krishnamurty, C.M. Reaves, S.P. Denbaars, P.M. Petroff, Appl. Phys. Lett. 63, 3203 (1993)

[2] J. Tersoff, C. Teichert, M.G. Lagally, Phys. Rev. Lett. 76, 1675 (1996).

[3] F. Liu, S.E. Davenport, H.M. Evans, M.G. Lagally, Phys. Rev. Lett. 82, 2528 (1999)

[4] Q. Xie, A. Madhukar, P. Chen, N. Kobayashi, Phys. Rev. Lett. 75, 2542 (1995).

[5] P. Schittenhelm, C. Engel, F. Findeis, G. Abstreiter, A.A. Darhuber, G. Bauer, A.O. Kosogov, P. Werner, J. Vac. Sci. Technol. B 16, 1575 (1998).

[6] G. Springholz, V. Holy, M. Pinczolits, G. Bauer, Science 282, 734 (1998).

[7] V. Holy, G. Springholz, M. Pinczolits, G. Baner, Phys. Rev. Lett. 83, 356 (1999). 
[8] G. Springholz, M. Pinczolits, P. Mayer, V. Holý, G. Bauer, H.H. Kang, L. Salamanca-Riba, Phys. Rev. Lett. 84, 4469 (2000).

[9] M. Strassburg, V. Kutzer, V.W. Pohl, A. Hoffmann, I. Broser, N.N. Ledentsov, D. Bimberg, A. Rosenauer, U. Fischer, D. Gehrtsen, I.L. Krestnikov, M.V. Maximov, P.S. Kop'ev, Z.I. Alferov, Appl. Phys. Lett. 72, 942 (1998).

[10] V. Holy, C. Giannini, L. Tapfer, T. Marschner, W. Stolz, Phys. Rev. B 55, 9960 (1997).

[11] A.A. Darhuber, J. Zhu, V. Holy, J. Stangl, P. Mikulik, K. Brunner, G. Abstreiter, G. Bauer, Appl. Phys. Lett. 73, 1535 (1998).

[12] C. Giannini, T. Baumbach, D. Luebbert, R. Felici, L. Tapfer, T. Marschner, W. Stolz, N.Y. Jin-Philipp, F. Philipp, Phys. Rev. B 61, 2173 (2000).

[13] H. Omi, T. Ogino, Appl. Phys. Lett. 71, 2163 (1997).

[14] J. Zhu, K. Brunner, G. Abstreiter, Appl. Phys. Lett. 73, 620 (1998).

[15] F. Liu, J. Tersoff, M.G. Lagally, Phys. Rev. Lett. 80, 1268 (1998).

[16] K. Brunner, J. Zhu, C. Miesner, G. Abstreiter, O. Kienzle, F. Ernst, Physica E 7, $881(2000)$.

[17] M. Schmidbauer, Th. Wiebach, H. Raidt, M. Hanke, R. Koehler, H. Wawra, Phys. Rev. B 58, 10523 (1998).

[18] S.K. Sinha, E.B. Sirota, S. Garoff, H.B. Stanley, Phys. Rev. B 38, 2297 (1988).

[19] V. Holy, U. Pietsch, T. Baumbach, High Resolution X-Ray Scattering from Thin Layers and Multilayers, Springer Tracts in Modern Physics, Vol. 149, Springer, Berlin 1999.

[20] M. Pinczolits, G. Springholz, G. Bauer, Phys. Rev. B 60, 11524 (1999).

[21] V. Holy, J. Stangl, G. Springholz, M. Pinczolits, G. Bauer, Mater. Res. Soc. Symp. Proc. 618, 161 (2001).

[22] V. Holy, T. Roch, J. Stangl, A. Daniel, G. Bauer, T.H. Metzger, Y.H. Zhu, K. Brunner, G. Abstreiter, Phys. Rev. B 63, 205318 (2001). 